1 Estimation of the Atmospheric Boundary Layer height during 2 different atmospheric conditions: A comparison on reliability of 3 several methods applied to lidar measurements

5 Toledo, D. ${ }^{1,2}$, Córdoba-Jabonero, C. ${ }^{1}$, Adame, J. A. ${ }^{1}$, De la Morena, B. ${ }^{1}$, 6 Gil-Ojeda, M. ${ }^{1}$

7

$8{ }^{1}$ Instituto Nacional de Técnica Aeroespacial (INTA), Atmospheric Research and 9 Instrumentation Branch, Torrejón de Ardoz, 28850-Madrid, Spain,

$10{ }^{2}$ Department of Physics, University of Oxford, Parks Rd, Oxford OX1 3PU, UK.

12 Corresponding Author: Carmen Córdoba-Jabonero, Instituto Nacional de Técnica 13 Aeroespacial (INTA), Ctra. Ajalvir km.4, Torrejón de Ardoz-28850, Madrid, Spain. 14 Phone/fax: +34 915201294/+34 915201633. E-mail: cordobajc@inta.es 


\section{Estimation of the Atmospheric Boundary Layer height during \\ 17 different atmospheric conditions: A comparison on reliability of several methods applied to lidar measurements}

The performance of six numerical methods usually used to determine the Atmospheric Boundary Layer (ABL) height from lidar measurements was investigated under different atmospheric conditions: results were compared with those obtained from radiosoundings to analyse their reliability for ABL-height retrievals. The selected methods were the gradient method (GM), the logarithm gradient method (LGM), the inflection point method (IPM), the wavelet covariance transform (WCT), the centroid/variance method (VM) and the cluster analysis (CA). Lidar measurements were carried out in the frame of the "Atmospheric Minor Species relevant to the Ozone Chemistry" (AMISOC) project during a multi-instrument campaign conducted at the INTA/Atmospheric Observatory "El Arenosillo" (INTA/ARN) in south-western Spain from 15 May to 20 June 2012. The goal of this work is to analyse the performance and robustness of the different lidar methods in this region, characterized by particular atmospheric conditions. In particular, both events of sealand breeze regimes and episodes of Saharan dust intrusions were studied. In most days, similar results were obtained by all lidar methods in the events of sea-land breeze regimes, presenting relative absolute differences between lidar and radiosounding retrievals below $12 \%$ in average. However, big discrepancies between lidar and radiosounding retrievals are found when residual layers are present in the measurements. In such cases, the vertical extension of lidar and radiosounding profiles must to be limited to the altitude of the residual layer bottom.

In a second analysis, focused on diurnal variability in the ABL heights under non-dusty (ND) and dust (DD) conditions, the methods were tested against intensive radiosoundings launched every 4 hours over 2 
days. Under ND conditions, the best results were achieved for the LGM, presenting a mean of the relative absolute differences respect to radiosounding measurements of $10 \%$. The rest of methods also provided good results with relative differences below $20 \%$ in average. Under DD conditions, however, an increase of the relative differences is found with mean values of up $32 \%$. In this case, best results are given by $\mathrm{CA}$ with a mean relative difference of $20 \%$. Despite the limited data set used in this work, results show that unlike the ND conditions for which all lidar methods provide good results respect to radiosounding retrievals, under DD conditions the election of the lidar method is a key factor for ABL estimation. However, we remark the need of extending our analysis to longer periods of time to better characterize the differences observed in this work.

Keywords: ABL; numerical methods; sea-land breeze regimes; lidar; radiosoundings; Saharan dust.

\section{Introduction}

The Atmospheric Boundary Layer (ABL) is defined as the lowest part of the troposphere that is directly influenced by the Earth's surface on a timescale of one hour or less (Stull, 1988). The ABL is affected by orography, land cover, weather conditions, and solar radiation in particular; thus, the layer evolves throughout the day. After sunny and clear sky conditions over land, the formation of stable layers (thermal inversions) is typical as the ground cools overnight. In the daytime, surface heating drives convective processes that generate unstable conditions (forming a mixing layer). Air pollutants are released into the ABL, where they mix with upper-level air via dispersion processes, according to the ABL structure and turbulence. Therefore, robust knowledge of ABL dynamics is crucial for weather forecast modeling and air pollution studies (Seibert et 
al., 1998, 2000).

Many different instruments and techniques have been used to estimate $\mathrm{ABL}$ heights. Radio soundings are the most reliable instruments because they use both potential temperature and humidity (Heffter 1980; Stull 1988). Unfortunately, the daily schedule of radiosonde launchings is, at most, twice per day. Hence, alternative instrumentation must be used on a daily basis. Active remote sensing systems, such as lidars, have been used to estimate ABL heights. Lidars offer two main advantages: a) the ability to detect the large aerosol gradients in the atmosphere that delimit the ABL and the Free Troposphere (FT), i.e., identification of the ABL top height; and b) the ability to operate on a continuous mode with a high temporal resolution.

Two types of methods have been used to determine the top of the ABL: 1) methods that find a sharp spike in the lidar signal in the transition zone (TZ) between the ABL and the FT; and 2) methods based on statistical analysis. The methods that use

90 the first approach assume that the aerosol concentration at heights below the ABL top is 91 significantly higher than that in the FT. For instance, Melfi et al. (1985) and Boers et al. 92 (1988) defined the ABL top as the height where the backscattered lidar signal drops below a given threshold. Hence, these methods have the disadvantage of requiring an appropriate selection of this threshold value, which depends on the lidar system and on 95 the weather conditions. For that reason, they are discarded in this work.

The most common procedure used to detect the ABL height in the backscattered lidar signal profile is an analysis of its derivative. There are three ways to analyze the derivative of the lidar signal with respect to height: the simple derivative (Hayden et al., 
100 1997; Flamant et al., 1997), the derivative of the logarithm of the signal (Senff et al., 101 1996), and the second derivative (Menut et al., 1999). These methods are good 102 candidates for estimating the ABL top height because the total time processing to

103 retrieve the heights is very low. However, the methods are affected by noise, and small-

104 scale structures in lidar signals introduce large gradients unrelated to the ABL top 105 height. Indeed, results obtained by derivative methods strongly depend on the value of 106 the lidar signal gradient at a given point.

107

The wavelet covariance transform (WCT) approach is less affected by the signal 109 noise and is also used for ABL top determination (Steyn et al., 1999; Cohn and 110 Angevine, 2000; Davis et al., 2000; Hägeli et al., 2000; Brooks, 2003). WCT, also

111 called Haar wavelet transform, provides a multi-scale local gradient analysis of the lidar 112 signal to isolate the spikes due to aerosol concentrations. A recently published 113 comparative study (Pal et al., 2010) shows that the ABL height obtained by the Haar 114 wavelet method ranges between the values estimated by the derivative of the logarithm 115 of the signal and the second derivative. Notably, these methods assume that the 116 transition range in the backscattered lidar signal from the ABL to the FT closely 117 corresponds to the temperature inversion zone (Brooks, 2003).

119 However, methods based on statistical analysis are related to large temporal 120 variability in the aerosol concentration in the $\mathrm{TZ}$ because clear air masses from the FT 121 are mixed with those from the ABL. A more widely applied method for ABL estimation 122 is the centroid/variance method (VM) (Hooper and Eloranta, 1986; Piironen and 123 Eloranta, 1995; Menut et al., 1999). This approach estimates the ABL height by using 124 the variance of the lidar signal over a particular time period. As a result, a high 
125 variability in the backscattered lidar signal in the $\mathrm{TZ}$ reveals a local maximum in the 126 variance profile at that height.

Additionally, cluster analysis (CA) is a method used for lidar-derived ABL

129 height estimations (see details in Toledo et al., 2014); the method combines both of the

130 previous approaches. CA is based on assembling a set of objects into groups

131 (Anderberg, 1973), where the objects in the same cluster are similar and objects in 132 different clusters are dissimilar (Balling, 1984). Hence, the ABL top height is identified

133 by analyzing the vertical distribution of the different clusters (Toledo et al., 2014).

In this work we compare $\mathrm{ABL}$ estimated from lidar measurements using the methods mentioned above with those obtained from radiosounding measurements under the particular atmospheric conditions found in the INTA/Atmospheric Observatory "E1 Arenosillo"; (1) events of sea-land breeze regimes; (2) dust intrusion events. The goal of this comparison is to study the performance of the different lidar methods under these

140 atmospheric conditions that directly influence on ABL.

\section{Methodology}

\subsection{AMISOC-ARN campaign: area description and lidar measurements}

In the framework of the "Atmospheric Minor Species relevant to the Ozone

147 Chemistry at both sides of the jet" (AMISOC) project, lidar measurements were 148 performed during a multi-instrument campaign conducted at the "El Arenosillo" 149 Atmospheric Observatory (ARN, $37.1^{\circ} \mathrm{N} 6.7^{\circ} \mathrm{W}, 40 \mathrm{~m}$ a.s.1.), which is managed by the 
150 Instituto Nacional de Técnica Aeroespacial (INTA). In particular, one of the objectives

151 of that project is to evaluate the impact of the ABL on surface ozone levels under dusty 152 conditions (Adame et al., 2015). Hence, ABL height estimation is specifically addressed

153 within the AMISOC project.

INTA temporarily deployed a Micro Pulse Lidar v.3 (MPL-3) system at the ARN station from 20 May to 15 June 2012 to measure ABL heights. The ARN observatory is located in a rural environment, the Doñana National Park, in the southwestern Iberian Peninsula; it is approximately $1 \mathrm{~km}$ from the Atlantic Ocean. Air masses originate from various regions, such as the Atlantic Ocean, Europe and African

160 deserts, and often cross this area. Thus, the ABL can be studied for a wide range of atmospheric conditions. In this work, dust intrusions in the area during AMISOC-ARN are specifically examined. Lidar v.3 (MPL-3) usually in routine operation within the NASA Micro Pulse Lidar NETwork (MPLNET, http://mplnet.gsfc.nasa.gov) at the Santa Cruz de Tenerife site

167 (Canary Islands, Spain), managed in collaboration with AEMET (Spanish Meteorological Agency). The system is a single-wavelength (523 nm), high-repetition $(2500 \mathrm{~Hz})$, low-power $(\sim 7 \mu \mathrm{J})$, eye-safe backscatter lidar that is commercially available,

170 and capable of determining the range of aerosols and clouds in a fully unattended 171 operation mode. The raw signals were corrected for several factors (Campbell et al., 172 2002) that affect the instrument. A more detailed description of this system and 173 corrections can be found in Cordoba-Jabonero et al. (2011). In particular, the full 174 overlap of the system is reached at around $3 \mathrm{~km}$ height, with uncertainty values of 10 
$17530 \%$ from $300 \mathrm{~m}$ height up, increased up to $60 \%$ at lower altitudes. Hence, data below

$176300 \mathrm{~m}$ height a.g.l. are usually disregarded in lidar inversion procedures to obtain

177 optical properties of aerosols. However, the ABL retrievals (see next Sect 2.2) are

178 applied to the lidar range-corrected signals (RCS) (no other lidar inversions are needed

179 for ABL top height determination) from ground level up. MPL-3 measurements were

180 performed on a daily basis, with a 1-min integration time and a vertical resolution of 15

$181 \mathrm{~m}$. These 1-min lidar RCS profiles were averaged over 10 minutes for both the variance

182 calculations and signal-to-noise $(\mathrm{s} / \mathrm{n})$ ratio enhancement; thus, 6 RCS profiles were

183 obtained for each hour (144 RCS profiles per day).

\subsection{Methods applied to lidar measurements for ABL height determination}

Since the methods used in this work are well known, only a brief description is presented. The methods used for ABL height estimation are the gradient method (GM), the logarithm gradient method (LGM), the inflection point method (IPM), the wavelet covariance transform (WCT), the centroid/variance method (VM) and the cluster analysis (CA). The ABL height is defined by GM and LGM as the altitude where is found the negative minimum of the first derivative of the RCS and the negative minimum of the first derivative of the RCS logarithm, respectively. For the IPM, the altitude is given where the curvature of the lidar RCS experiences a sign change. In

195 particular, when applying the IPM, Menut et al. (1999) used the absolute minimum of

196 the second derivative of the RCS, whereas Sicard et al. (2006) defined the ABL top as 197 the altitude of the minimum found below the ABL height retrieved by GM. wavelet function, particularly the Haar function (see, e.g., Brooks, 2003), depending on 
200 the translation and dilation parameters. Translation defines the height of the Haar 201 function, and dilation represents the vertical extent of the function. Hence, the ABL top 202 height is located where the maximum WCT value is found by applying appropriate

203 values of those two parameters, depending on the depth of the TZ. Signal noise 204 dominates the vertical WCT profiles for low dilation values; alternatively, the WCT 205 maximum represents the ABL height for dilation values close to the depth of the TZ 206 between the ABL and the FT.

207 The last two methods, VM and CA, are based on statistical analysis to define the 208 ABL height. Based on the high temporal variability of the aerosol concentration in the $209 \mathrm{TZ}$, the ABL height is identified by $\mathrm{VM}$ as the lowest height position of a local 210 maximum in the temporal variance profile of RCS. The CA method is based on the 211 assembling a set of objects into groups (Anderberg, 1973), where the objects in the 212 same cluster are similar and objects in different clusters are dissimilar (Balling, 1984).

213 In particular, the distance between objects is the measure of the similarity between 214 clusters. These distances are based on single or multiple dimensions, where each 215 dimension represents a condition that groups the objects. Each dimension is represented 216 by the height, the lidar RCS and the variance for a given period. Clusters can be formed 217 using these distances with normalized values of all the variables. Therefore, the 218 distribution of the aerosols in the atmosphere and variations in their concentrations can 219 be studied simultaneously using this method. The ABL top height is defined as the 220 height where a change from one cluster to another is observed (Toledo et al., 2014).

\subsection{Meteorological observations: surface and profiles}


225 Temperature, relative humidity, pressure, rainfall, wind speed and wind direction data were registered by Vaisala 520WXT sensors located at three heights $(10,50$ and $100 \mathrm{~m}$ a.g.1.) on a 100-m high tower. In addition, meteorological profiles were obtained by using Vaisala radiosonde launches. Vertical measurements of temperature, pressure, humidity and wind (direction and speed) were registered with an accuracy/resolution of $<0.2^{\circ} \mathrm{C} / 0.1^{\circ} \mathrm{C},<0.6 \mathrm{hPa} / 0.1 \mathrm{hPa}$, and $<5 \% / 1 \%$, respectively. The uncertainty in the wind speed and direction is $0.15 \mathrm{~m} \mathrm{~s}^{-1}$ and 2 degrees, respectively. All the soundings experienced a mean flight time of approximately 80 minutes and reached an altitude of approximately $22-23 \mathrm{~km}$.

A total of 26 radiosoundings were launched on a daily basis at approximately 11:00 UTC. Additionally, two intensive launching schedules were planned for analyzing the robustness of the different methods throughout the day in relation to Saharan dust conditions. Non-dusty (ND) and dusty (DD) conditions were observed on 23-24 May and 31 May-1 June 2012, respectively. The criterion to identify the DD

240 conditions, as adopted from Córdoba-Jabonero et al. (2011), is based on AERONET

241 (AErosol Robotic NETwork, www.aeronet.nasa.gov) parameters: DD conditions are 242 fulfilled for threshold Aerosol Optical Depth (AOD) values higher than 0.2 and 243 Angstrom Exponents (AEx) lower than 0.5 (predominance of coarse particles). 244 Otherwise, the present conditions correspond to a ND case. The AOD for the DD period 245 ranges from 0.24 to 0.43 , and with AEx values below 0.5, while for the ND period we 246 found AODs less than 0.15 and AEx values greater than 0.9 (Adame et al., 2015). In 247 both intensive 2-day periods, the first radiosounding was launched at approximately 248 11:00 UTC, followed by 5 radiosoundings every 4 hours. In general, the sunshine 
duration is approximately from 05:30 UTC (sunrise) to 19:30 UTC (sunset); hence, convective mixing in the ABL becomes relevant during this period.

Depending on convective conditions, two different approaches were used to estimate the $\mathrm{ABL}$ height from the radiosounding measurements. Under convective conditions, the ABL top is obtained by averaging the heights estimated from both the potential temperature and specific humidity profiles. The ABL top is defined as the height where the maximal/minimal vertical gradient of the potential temperature/specific humidity is found (Oke, 1988; Sorbjan, 1989; Garratt, 1992). Above this altitude, the atmosphere can be considered the Free Troposphere (FT). Under surface inversion conditions, the ABL height is identified as the maximum height 260 of the surface inversion layer.

To determine the robustness of each method, the ABL heights are calculated by 263 all the proposed methods (GM, VM, WCT, LGM, IPM and CA) and are compared with 264 those obtained from the daily radiosoundings. Hence, the absolute differences between 265 the values obtained by each method and by the radiosounding retrievals are presented in terms of their mean and one-standard-deviation $(1 \sigma)$ values. Those differences were

267 taken in absolute value in order to avoid negative values in the calculation of the mean.

268 In addition, we did not observe in our study any lidar method providing systematically 269 ABL altitudes above or below radiosunding retrievals. The confidence of each method 270 in retrieving the ABL height under different atmospheric conditions is also analyzed in 271 specific situations: 1) particular sea-land breeze conditions in which the ABL evolution 272 is determined during the AMISOC-ARN campaign with respect to discrete daily 273 radiosoundings (launched at around 11 UTC); and 2) dissimilar aerosol scenarios under 
274 DD and ND conditions in which the ABL evolution during the day (day/night 275 variations) is determined with respect to intensive radiosoundings (launched every 4 276 hours for two consecutive days).

\section{Results and discussion}

3.1 ABL evolution during the AMISOC-ARN campaign: identification of sea-land breeze conditions governed by synoptic flows for only three days $(\sim 10 \%$ of the total campaign period) and by mesoscale processes for the remaining period. Two well-known sea-land breeze patterns usually occur in this area, classified as pure and non-pure. Pure breezes occur with flows (diurnal and nocturnal) perpendicular to the coast line, whereas non-pure breezes occur under synoptic forcing conditions (nocturnal) that not quite perpendicular to the coast (Adame et al., 2010). According to this criterion, 12 days were identified as pure breezes and 14 days as non-pure breezes. Figure 1 shows the surface weather conditions (temperature, relative humidity and wind) for pure (22 to 25 May 2012) and non-pure (6 to 8 June 2012) conditions.

FIGURE 1

Pure breezes reflect flows perpendicular to the coastline and well-established

297 daily cycles of temperature and humidity. An increase in the temperature is observed 298 each day due to the recirculation of the same air. Non-pure breezes exhibit nocturnal 
299 flows that are not perpendicular to the coastline and that are associated with synoptic

300 forcing conditions (higher wind speeds than pure breeze regimes); in this case, the daily

301 cycles of temperature and relative humidity are not clear.

302

The ABL heights obtained using each method and from the radiosoundings as well as the absolute differences (absDiff) between the ABL height values calculated using the six methods and those obtained from radiosoundings are shown in Figure 2; the error bars represent the standard deviations of the mean ABL top heights calculated by each method from the ten 1-min lidar profiles used in the averaging procedure. The bands limited by dashed lines represent the corresponding breeze regime identified for each day to distinguish pure (indicated by double arrows) and non-pure cases (no

310 arrows). Since the comparison between both instruments could be affected by the velocity and direction of the wind a correlation analysis between these parameters and the absolute and relative differences between the radiosounding and lidar retrievals was performed. In this analysis we did not found an increase of such differences for the days

314 with a higher wind speed (maxima wind speed around $9 \mathrm{~ms}^{-1}$ ) or for the days with a

315 wind direction near $220^{\circ} \mathrm{N}$ (angle between the North and the line perpendicular to the coastline). Therefore, balloon drifts do not noticeably affect the comparison illustrated in Figure 2. The temporal resolution of radiosounding measurements was of 5 seconds, resulting in a vertical resolution between 12 and $34 \mathrm{~m}$ at altitudes near ABL height.

\section{FIGURE 2}


324 pure and non-pure breezes, respectively. Non-pure breeze conditions are developed with

325 lower surface temperatures and higher wind speeds than pure breezes with synoptic 326 forcing at the upper levels (Hernandez-Ceballos, et al., 2013). These conditions are less

327 favorable for the development of convective processes; lower mixing heights are 328 observed in non-pure cases than in pure cases. In most days, the ABL height was 329 retrieved under cloud or residual layer free conditions for which all lidar methods and 330 radiosounding measurements provided similar altitudes. An example of one of those 331 days is illustrated in Figure 3 that shows the time variation of lidar RCS on 21 May 332 2012, the ABL height estimated by GM and VM, and the radiosounding ABL height 333 estimated for that day. We can observe that both lidar methods are in agreement with 334 differences below $100 \mathrm{~m}$ in most ABL retrievals (similar results were found for the rest 335 of lidar methods). However, Figure 2 shows big deviations between VM and the rest of 336 retrievals for the days 24-25 May, 29 May and 12 June. These differences are caused by 337 the presence of residual layers during the time period when the radiosounding was 338 launched. An example is illustrated in Figure 4, where the white arrow indicates a 339 residual layer between 9 UTC and 11:40 UTC. In this case, we can observe that GM 340 and VM provide similar ABL altitudes except between 11 UTC and 11:40 UTC (similar 341 differences are also found between VM and the rest of lidar methods). The main reason 342 of such differences is the fact that all lidar methods except VM provide the altitude of 343 the residual layer. As observed in Figure 4, at around 11:00 UTC the bottom of the 344 residual layer lies just above the ABL height. At that moment, all lidar methods except 345 VM provide the top height of the residual layer. Radiosounding measurements also 346 provide the altitude of the residual layer as result of its humidity gradient. However,

347 VM estimates correctly the altitude of the growing ABL, being therefore the most 348 reliable method in this analysis when residual layers are present. 
FIGURE 3

\section{FIGURE 4}

Excluding these days, similar estimates are derived by all lidar methods. Among

354 the pure-breeze cases, the smallest difference is found for the IPM with a value below $35515 \mathrm{~m}$ and the largest difference is found for the VM with a value of about $350 \mathrm{~m}$.

356 However, the smallest absDiff maximum is found for WCT with a value of $197 \mathrm{~m}$. This

357 feature is very important to analyze for evaluating the stability of each method because 358 the deviation between each method and radiosounding retrievals is highly dominated by 359 the maximum absDiff value. Under non-pure breeze conditions, almost all of the 360 methods present small differences in ABL top heights with respect to the heights 361 retrieved by the radiosoundings. In particular, the smallest and largest absDiff values 362 are obtained for WCT, respectively (see Fig. 2b, bottom panel). The smallest maximum 363 absDiff value is obtained for CA $(111 \mathrm{~m})$. The ABL top heights derived by the VM 364 under non-pure breeze conditions are consistent with the radiosounding retrievals. In 365 particular, the smallest absDiff value $(148 \mathrm{~m})$ is found on 3 June by the VM, whereas a 366 value greater than $270 \mathrm{~m}$ is obtained for the other methods.

The mean absolute relative difference (mean reDiff), mean absDiff and the one standard deviation $(1 \sigma)$ obtained by each method for all the pure and non-pure breeze cases, as well as for the overall period, are shown in Figure 5 (excluding the days 24-25 May, 29 May and 12 June). These results represent the robustness of the different methods in determining the ABL top height. 
377 the mean absDiff and reDiff, whereas the VM reports the highest mean absDiff $(>136$

$378 \mathrm{~m}$ ) with a mean reDiff of 0.09 . Low mean absDiff and reDiff values are also found for 379 CA, $116 \mathrm{~m}$ and 0.089 , respectively. Among the derivative methods, best results are 380 given by LGM with mean absDiff and reDiff values of $103 \mathrm{~m}$ and 0.08 . This result can 381 clearly be explained by the enhancement of the aerosol contrast obtained by taking the 382 logarithm of the RCS lidar signal. By examining the $1 \sigma$ values, the WCT shows the 383 smallest value. However, the other methods are reasonably consistent according to their 384 mean absDiff values. In general, all methods are in agreement with the radiosounding 385 retrievals for this period with mean absDiff and reDiff values below $137 \mathrm{~m}$ and 0.095 , respectively; the best results are obtained for WCT and LGM. The results obtained for non-pure breeze cases using the GM, LGM, IPM, VM, WCT and CA are very similar to those found under pure breeze conditions with mean absDiff and reDiff values below $130 \mathrm{~m}$ and 0.11 , respectively. Specifically, a mean absDiff value lower than $100 \mathrm{~m}$ is obtained for the VM, whereas a value of $130 \mathrm{~m}$ is found for the IPM. In this period best results are given by VM although the differences in the mean absDiff respect to the other methods are below $30 \mathrm{~m}$. By analyzing the

394 overall period (third panel of Figure 5), the ABL altitude retrieved by lidar methods are 395 in good agreement with radiosounding estimations. The best results are obtained by 396 LGM with a mean absDiff value of $107 \mathrm{~m}$. However, we remark that the rest of 397 methods provide mean absDiff values below $128 \mathrm{~m}$. In summary we found that all lidar 398 methods provided similar results with differences below $40 \mathrm{~m}$ in average. However, 
399 limitations are found when residual layers are present in the measurements. Under such

400 conditions, $\mathrm{VM}$ provides the most reliable ABL retrievals.

401

402

\subsection{ABL evolution during the day: influence of the aerosol conditions}

403

Two aerosol conditions were identified and selected during the AMISOC-ARN campaign by intensive radiosonde launchings: 24-25 May 2012 and 31 May-01 June 2012 under non-dusty (ND) and dusty (DD) conditions, respectively. Both periods

featured pure-breeze flow regimes most of the time. For the first intensive period, a total of 7 radiosoundings were launched every 4 hours. Because of the low ABL height value obtained for the radiosounding launched at 04:00 UTC, this case is not included. In the

410 second intensive period, a total of 6 radiosoundings were launched every 4 hours

411 (unfortunately, the last radio sounding launched at approximately noon failed).

412

As mentioned in section 3.1, residual layers were present for the days 24-25

414 May, resulting in vertical or temporal lidar signal gradients at altitudes above the ABL

415 height. In this regard, we found necessary to limit the top of lidar and radiosounding 416 profiles to altitudes below the bottom of the residual layers. The ABL heights obtained 417 by the six methods and those retrieved from the radiosounding data during the ND and DD periods are shown in Figures 6a and 7a, respectively.

FIGURE 6

FIGURE 7 
only under convective conditions; however, only the first radiosounding in the DD

425 period experienced these conditions. For these cases, the ABL height values were

426 higher than those for the rest of the intensive period, as expected for atmospheric

427 convection. The error obtained during both periods for the different methods decreased

428 as the convective conditions diminished, likely associated with more nocturnal stability.

429 Indeed, under these conditions, the ABL presents a strong static stability; hence, its

430 vertical variability over time is very low. Although all of the methods well reproduce

431 the behavior of the ABL evolution during the day, differences are found with respect to

432 the radiosounding retrievals.

In order to examine these discrepancies, the absolute differences (absDiff) 435 between the ABL heights calculated using each method and those obtained from the 436 radiosoundings under the ND and DD conditions are shown in Figures $6 \mathrm{~b}$ and $7 \mathrm{~b}$. The 437 smallest absDiff values are obtained for the ND period by the GM, LGM, VM and CA 438 with values lower than $15 \mathrm{~m}$, whereas the largest absDiff values correspond to the WCT 439 (around $200 \mathrm{~m}$ ). The smallest maximum absDiff value is found for IPM $(117 \mathrm{~m})$. In 440 comparison with the results obtained during the discrete daily radiosounding period (see 441 Sect. 3.1), similar results are found for the different lidar methods with absDiff values 442 below $150 \mathrm{~m}$ in most cases. Regarding the derivative methods, a good agreement is 443 found with respect to the radiosounding retrievals. During the DD intensive period (31 444 May-01 June 2012), the lowest and highest absDiff values are found by CA (11 m) and 445 the VM (182 m), respectively, and the smallest maximum is obtained by CA and WCT $446 \quad(126 \mathrm{~m})$. 
In order to study the robustness of each method, the mean of reDiff and absDiff,

449 and the corresponding $1 \sigma$ values for the ND and DD periods are shown in Figure 8. For

450 ND period, all methods provide reasonable mean values of reDiff and absDiff. In

451 particular, best results are given by LGM with a mean reDiff of 0.10 and a mean

452 absDiff of $58 \mathrm{~m}$. By constrast, the highest values of these parameters for this period is

453 given by WCT. For DD period, a remarkable increase of the mean values of reDiff and

454 absDiff is observed for the derivative methods and VM. The lowest values of the mean absDiff and mean reDiff are found for the CA method, $56 \mathrm{~m}$ and 0.20 , respectively.

In summary, results illustrated in Figures 6 and 8 show that for the ND period all lidar methods are in agreement with radiosounding retrievals with mean reDiff values below 0.21 . However, for DD period CA is the only method providing a mean reDiff below 0.21 (see Figures 7 and 8). As mentioned in section 2.1, the overlap uncertainties increase at altitudes below $300 \mathrm{~m}$. Therefore a similar analysis as that shown in Figure 8 was performed but considering only the cases with a ABL height above $300 \mathrm{~m}$. The results are illustrated in Figure 9 where again CA provides the best result for DD period.

464 A remarkable result is the noticeable decrease of mean reDiff values of the different lidar methods for the ND period. As reported by other authors (e.g. Pal, 2014), this decrease clearly shows that ABL height lidar retrievals are affected when overlap

467 uncertainties become relevant. For the DD period the decrease in mean reDiff is smaller 468 as result of the fewer number of cases of ABL heights below $300 \mathrm{~m}$. Although results in 469 Figure 8 indicate that lidar and radiosounding retrievals are in agreement, this analysis 470 points out the need of decrease overlap errors at altitudes below $300 \mathrm{~m}$ to better 471 characterize the ABL during nighttime. 


\section{Conclusions}

ABL heights were determined during the AMISOC-ARN campaign by applying six mathematical methods to lidar measurements: the gradient method (GM), the logarithm gradient method (LGM), the inflection point method (IPM), the wavelet covariance transform (WCT), the centroid/variance method (VM) and the cluster analysis (CA). In order to analyze the performance of these methods, the ABL height results were compared with those derived from potential temperature and specific humidity profiles from radiosoundings. comparison under different atmospheric conditions: 1) sea-land breeze conditions, in which the ABL evolution during the entire AMISOC-ARN campaign was determined; and 2) dissimilar aerosol conditions, i.e., under dusty (DD) and non-dusty (ND) conditions.

492 First, each method was tested against discrete daily radiosounding measurements during 493 the campaign for two types of coastal breeze regimes, and the convective conditions 494 present in each case were examined. Excluding the cases with presence of residual 495 layers, all methods provide similar results with relative absolute differences respect to 496 radiosounding retrievals below $12 \%$ in average. Despite that best results were given by 497 LGM, VM and WCT, the differences respect to the others methods were smaller than 
$40 \mathrm{~m}$ in average. Since the vertical resolution of lidar and radiosounding measurements

499 is of 15 and $34 \mathrm{~m}$ (maximum value found in this study), respectively, these results point 500 out the need of improving the accuracy of ABL height estimation from these

501 instruments to better analyze the differences observed in this period. Limitations of lidar 502 methods were found in days with residual layers present at the radiosounding launch 503 times. Under such conditions, we had to limit the vertical extension of lidar and 504 radiosounding profiles to the altitude of the residual layer bottom.

Second, all of the methods were tested against intensive radiosounding retrievals

507 under DD and ND conditions. Under ND conditions, best results are archived by GM, 508 LGM, IPM and CA, with mean values of relative absolute differences of $16 \%$.

509 However, the maxima difference in average between the different methods is of $35 \mathrm{~m}$.

510 Since the vertical resolution of radiosounding measurements ranges between 12 and 34

$511 \mathrm{~m}$, this result clearly shows the need of improve the vertical resolution of ABL height

512 measurements to investigate those differences. CA provides the best results under dusty 513 conditions with a relative difference of $20 \%$ in average. The rest of methods provide 514 mean values greater than $24 \%$.

In summary, under non-dusty conditions we found that all lidar methods are in 517 agreement with radiosounding measurements. Though differences in lidar ABL height 518 retrievals are found between the different methods, the vertical accuracy of the 519 measurements clearly limits the interpretation of those differences. In spite of these 520 limitations, results illustrated in Figures 5 indicate an increase in the differences under non-pure breeze conditions respect to the pure ones. A similar result is also observed in

522 Figure 8, where a noticeable increase of the differences is given under the dusty 
523 conditions. By comparing ABL altitudes under dusty and non-dusty conditions, we have

524 observed lower ABL heights when dust intrusion events are present, observing even 525 stable conditions in the early evening. Similarly, lower ABL altitudes are given under 526 non-pure breeze conditions respect to pure conditions as result of the lower 527 temperatures at surface. These increases in the differences indicate that the error in ABL 528 estimation (using lidar measurements) increases as the mixing altitude decreases.

529 However, we remark the need of extending this analysis carried out under these 530 different atmospheric conditions for longer periods of time.

\section{Acknowledgements}

534 This work was supported by the Spanish Ministerio de Economía y Competitividad 535 (MINECO) under grants CGL2011-24891 (AMISOC) and CGL2014-55230-R 536 (AVATAR). The authors especially thank the INTA/Atmospheric Observatory "El 537 Arenosillo" (ARN) staff for their valuable assistance and support in maintaining the 538 lidar system and launching the radiosoundings.

\section{$540 \quad$ References}

541 Adame, J. A., J. P. Bolívar, and B. de la Morena, 2010, Surface ozone measurements in

542 the southwest of the Iberian Peninsula (Huelva, Spain). Environ. Sci. Pollut. Res., 17, $543 \quad 355-368$.

544 Adame, J.A., C. Córdoba-Jabonero, M. Sorribas, D. Toledo, M. Gil-Ojeda, 2015, 545 Atmospheric boundary layer and ozone-aerosol interactions under Saharan intrusions 546 observed during AMISOC summer campaign. Atmos. Environ., 104, pp. 205-216.

547 Anderberg, M. R., 1973, Cluster Analysis for Applications. Academic Press, 359 pp. 
548 Balling, R. C., Jr., 1984, Classification in climatology. Spatial Statistics and Models, G.

549 L. Gaile and C. J. Willmott (Eds.), Reidel, 81-108.

550 Boers, R., J. D. Spinhirne, and W. D. Hart, 1988, Lidar Observations of the fine-scale

551 variability of marine stratocumulus clouds. J. App. Meteor., 27, 797-810.

552 Brooks, I., 2003, Finding Boundary Layer Top: Application of a wavelet covariance 553 transform to lidar backscatter profiles. J. Atmos. Ocean. Tech., 20, 1092-1105.

554 Campbell, J. R., D. L. Hlavka, E. J. Welton, C. J. Flynn, D. D. Turner, J. D. Spinhirne, 555 V. S. Scott III, and I. H. Hwang, 2002, Full-time, eye-safe cloud and aerosol lidar 556 observation at Atmospheric Radiation Measurement program sites: Instruments and data 557 processing. J. Atmos. Ocean. Tech., 19, 431-442.

558 Cohn, S. A., and W. M. Angevine, 2000, Boundary layer height and entrainment zone 559 thickness measured by lidars and wind-profiling radars. J. App. Meteor., 39, 1233-1247. 560 Córdoba-Jabonero, C., M. Sorribas, J. L. Guerrero-Rascado, J. A. Adame, Y. 561 Hernández, H. Lyamani, V. Cachorro, M. Gil, L. Alados-Arboledas, E. Cuevas, and B. 562 de la Morena, 2011, Synergetic monitoring of Saharan dust plumes and potential impact 563 on surface: a case study of dust transport from Canary Islands to Iberian Peninsula. 564 Atmos. Chem. Phys., 11, 3067-3091, doi:10.5194/acp-11-3067-2011.

565 Davis, K. J., N. Gamage, C. R. Hagelberg, C. Kiemle, D. H. Lenschow, and P. P. 566 Sullivan, 2000, An objective method for deriving atmospheric structure from airborne 567 lidar observations. J. Atmos. Ocean. Tech., 17, 1455-1468.

568 Flamant, C., J. Pelon, P. H. Flamant, and P. Durand, 1997, Lidar determination of the 569 entrainment zone thickness at the top of the unstable marine atmospheric boundary $570 \quad$ layer. Bound. Layer Meteor., 83, 247-284.

571 Garratt, J. R., 1992, The Atmospheric Boundary Layer. Cambridge Atmospheric and 572 Space Science Series, Cambridge Univ. Press, 335 pp. 
573 Hägeli, P., D. G. Steyn, and K. B. Strawbridge, 2000, Spatial and temporal variability of

574 mixed-layer depth and entrainment zone thickness. Bound. Layer Meteor., 97, 47-71.

575 Hayden, K. L., K. G. Anlauf, R. M. Hoff, J. W. Strapp, J. W. Bottenheim, H. A. Wiebe,

576 F. A. Froude, J. B. Martin, D. G. Steyn, and I. G. McKendry, 1997, The Vertical

577 Chemical and Meteorological Structure of the Boundary Layer in the Lower Fraser

578 Valley during Pacific '93. J. Atmos. Environ., 31, 2089-2105.

579 Heffter, J. L., 1980, Air Resource Laboratories atmospheric transport and dispersion 580 model. NOAA Tech. Memo. ERL ARL-81, 24 pp.

581 Hernández-Ceballos, M. A., J. A. Adame, J. P. Bolívar, and B. de la Morena, 2013, A

582 mesoscale simulation of coastal circulation in the Guadalquivir valley (southwestern

583 Iberian Peninsula) using the WRF-ARW model. Atmos. Res., 124, 1-20.

584 Hooper, W. P., and E. W. Eloranta, 1986, Lidar measurements of wind in the planetary 585 boundary layer: The method, accuracy and results from joint measurements from 586 radiosonde and kytoon. J. Clim. App. Meteor., 25, 990-1001.

587 Melfi, S. H., J. D. Spinhirne, S. H. Chou, and S. P. Palm, 1985, Lidar observation of the 588 vertically organized convection in the Planetary Boundary Layer over the ocean. $J$. 589 Clim. App. Meteor., 24, 806-821.

590 Menut, L., C. Flamant, J. Pelon, and P. H. Flamant, 1999, Urban boundary-layer height 591 determination from lidar measurements over the Paris area. App. Opt., 38, 945-954.

592 Oke, T. R., 1988, Boundary Layer Climates, 2nd ed., HalstedPress, New York, 435 pp.

593 Pal S., 2014, Monitoring Depth of Shallow Atmospheric Boundary Layer to 594 Complement LiDAR Measurements Affected by Partial Overlap. Remote Sensing, 6(9), $5958468-8493$.

596 Pal, S., A. Behrendt, and V. Wulfmeyer, 2010, Elastic-backscatter-lidar-based 597 characterization of the convective boundary layer and investigation of related statistics. 598 Ann. Geophys., 28, 825-847. 
599 Piironen, A K., and E. W. Eloranta, 1995, Convective boundary layer depths and cloud 600 geometrical properties obtained from volume imaging lidar data. J. Geophys. Res., 100, $601 \quad 569-576$.

602 Seibert, P., F. Beyrich, S. E. Gryning, S. Joffre, A. Rasmussen, and P. Tercier, 1998, 603 Mixing layer depth determination for dispersion modeling. In: COST Action 710 604 Final Report. Harmonization of the pre-processing of meteorological data for 605 atmospheric dispersion models, Report of Working Group 2, Office for Official 606 Publications of the European Communities, Luxembourg, $431 \mathrm{pp}$.

607 Seibert, P., F. Beyrich, S. E. Gryning, S. Joffre, A. Rasmussen, and P. Tercier, 2000, 608 Review and intercomparison of operational methods for the determination of the mixing 609 height. Atmos. Environ., 37, 1001-1027.

610 Senff, C., J. Bösenberg, G. Peters, and T. Schaberl, 1996, Remote sensing of turbulence 611 ozone fluxes and the ozone budget in the convective boundary layer with DIAL and 612 Radar-RASS: A case study. Contributions to Atmospheric Physics, 69, 161-176.

613 Sicard, M., C. Pérez, F. Rocadenboch, J. M. Baldasano, and D. García-Vizcaino, 2006, 614 Mixed layer depth determination in the Barcelona costal area from regular lidar 615 measurements: Methods, results and limitation. Bound. Layer Meteor., 119, 135-157.

616 Sorbjan, Z., 1989, Structure of the Atmospheric Boundary Layer. Prentice Hall, 617 Englewood Cliffs, N.J., 317 pp.

618 Steyn, D. G., M. Baldi, and R. M. Hoff, 1999, The detection of mixed layer depth and 619 entrainment zone thickness from lidar backscatter profiles. J. Atmos. Ocean. Tech., 16, $620 \quad 953-959$.

621 Stull, R. B., 1988, An introduction to boundary layer Meteorology. Kluwer Academic 622 Publishers, Dordrecht, Netherlands, 666 pp. 
623 Toledo, D., C. Córdoba-Jabonero, and M. Gil-Ojeda, 2014, Cluster Analysis: A new

624 approach applied to Lidar measurements for Atmospheric Boundary Layer height 625 estimation. J. Atmos. Ocean. Tech., 31 (2), 422-436.

626

627 

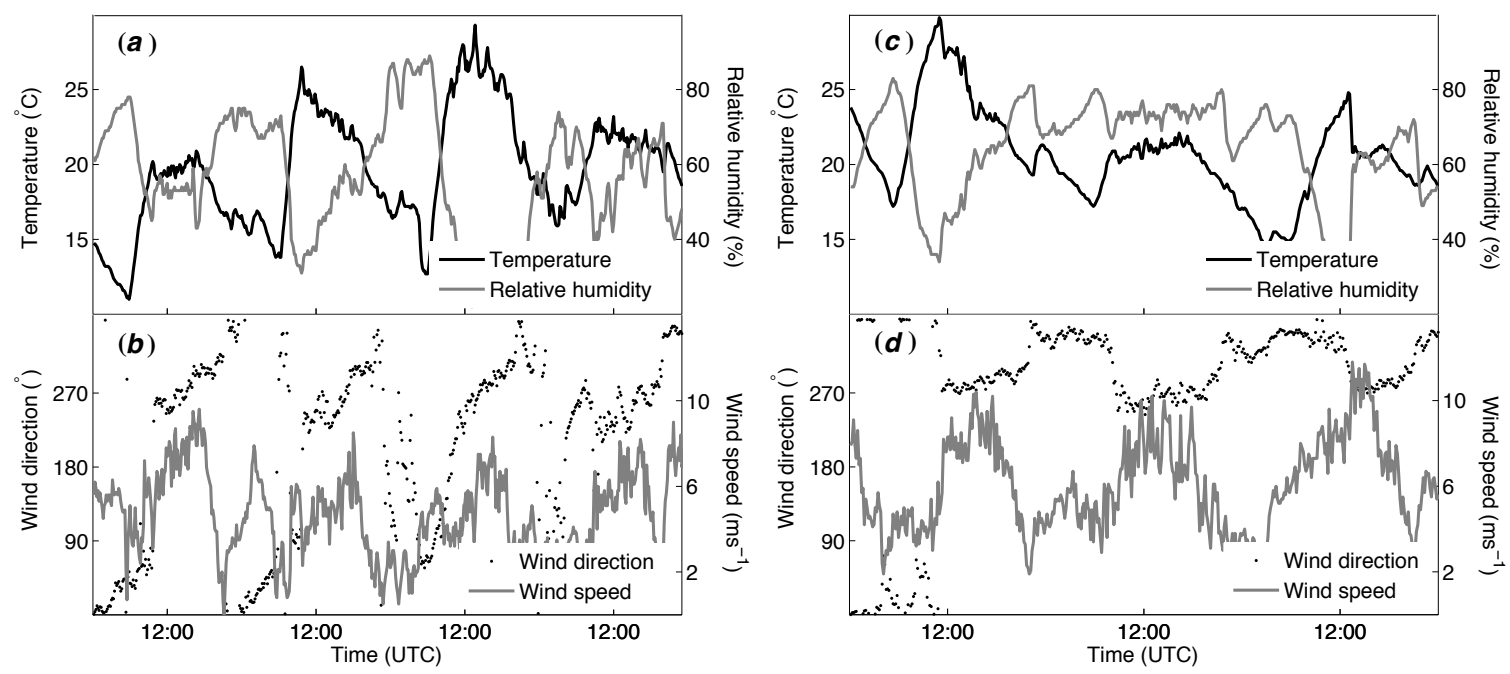

629 Figure 1. Surface evolution of the temperature and relative humidity and wind speed

630 and direction (bottom) for (a-b) pure breeze conditions from 22 to 25 May 2012, and (c-

631 d) non-pure breeze regimes from 06 to 08 June 2012.

632

633

634

635

636 


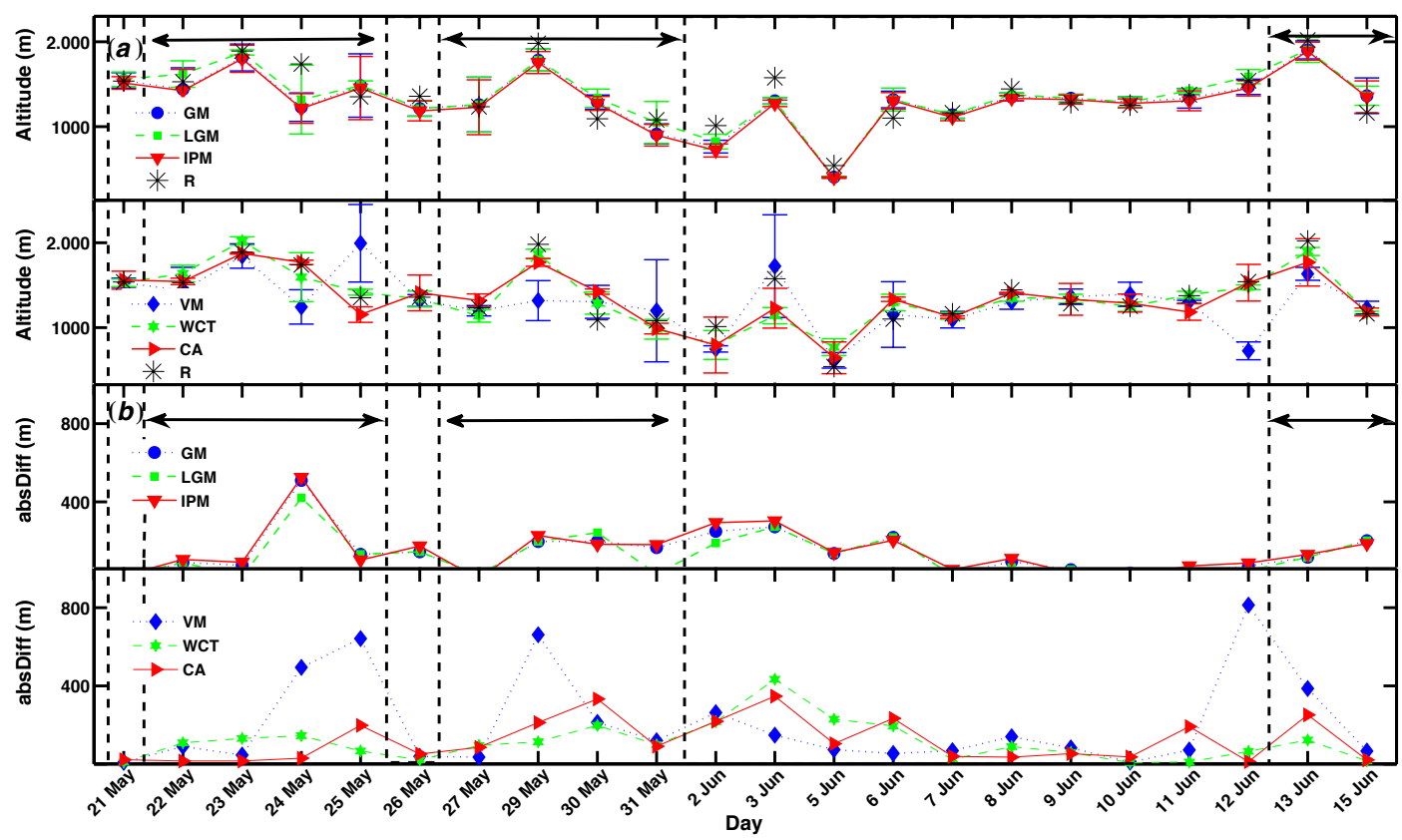

638 Figure 2. (a) ABL heights derived from the GM (gradient method), LGM (logarithm

639 gradient method), IPM (inflection point method), VM (centroid/variance method), WCT

640 (wavelet covariance transform), CA (cluster analysis) and the radiosounding retrieval

641 (R) during the overall AMISOC-ARN campaign period. (b) Absolute differences

642 (absDiff) between the ABL heights obtained by the radiosounding data and those

643 calculated using the six methods. The bands delimited by the dashed lines represent the

644 corresponding breeze regimes identified for each day to distinguish pure (indicated by

645 double arrows) and non-pure cases.

646

647

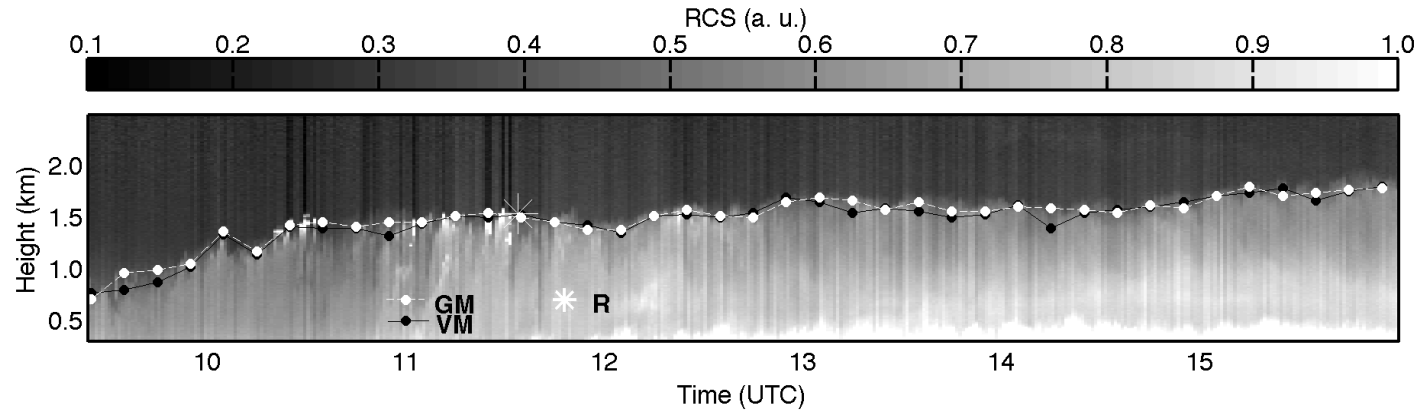

648 Figure 3. Temporal evolution of both the lidar RCS and the ABL top height as 


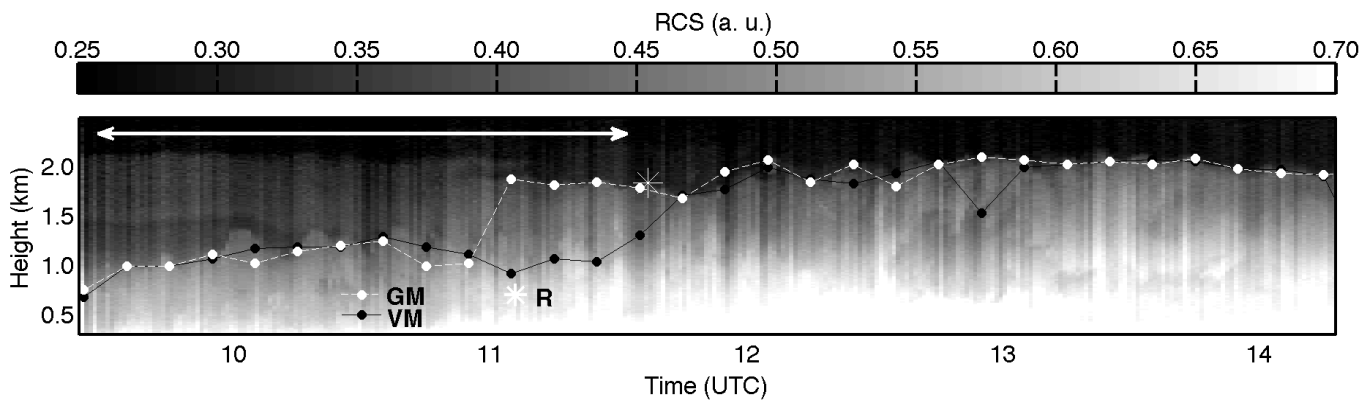

650

651 Figure 4. Same as figure Fig. 3 but on 29 May 2012. The white double arrow indicates

652 the time interval for which a residual layer is observed.

653

654

655

656

657

658

659

660

661

662

663

664

665

666

667

668

669 


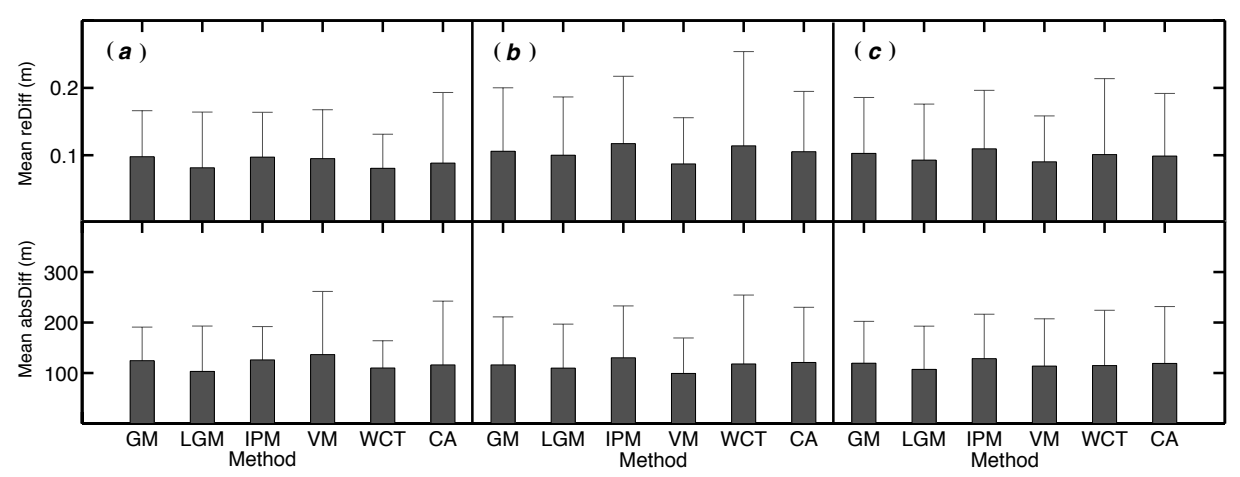

670

671 Figure 5. The mean absolute relative difference (mean reDiff) and mean absolute

672 difference (mean absDiff) values and corresponding one standard deviation ( $1 \sigma$, shown

673 by error bars) obtained by each method shown in Fig. 2 for (a) pure breeze cases, (b)

674 non-pure breeze cases, and (c) for the overall period.

675

676

677

678

679

680

681

682

683

684

685

686

687

688

689 
690
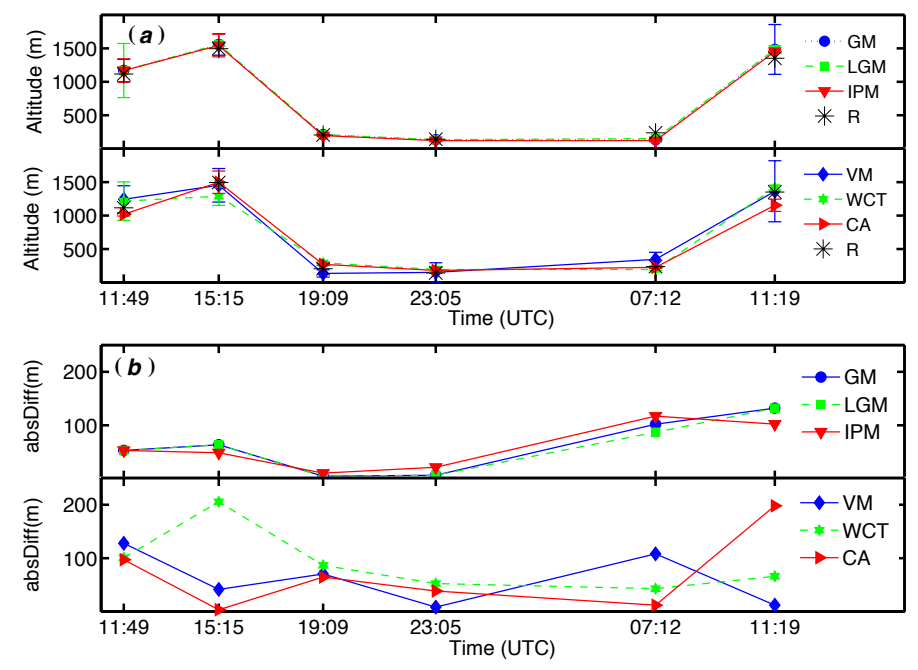

691 Figure 6. (a) and (b) are the same as Fig. 2a and $2 \mathrm{~b}$ of the manuscript, respectively, but

692 during the intensive radio soundings period corresponding to ND conditions.

693

694

695

696

697

698

699

700

701

702

703

704

705

706

707

708 


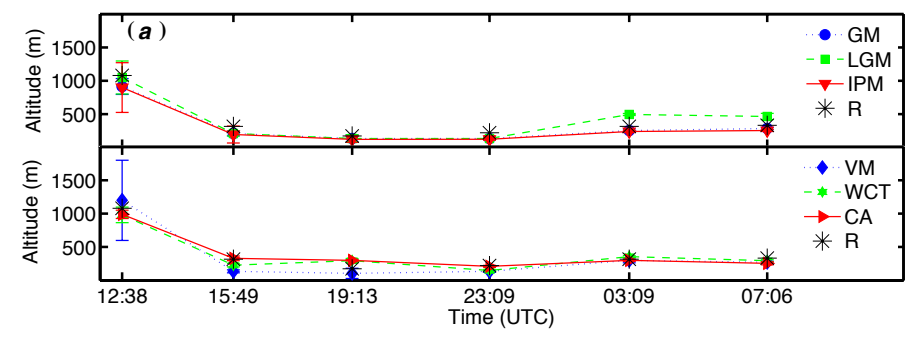

709

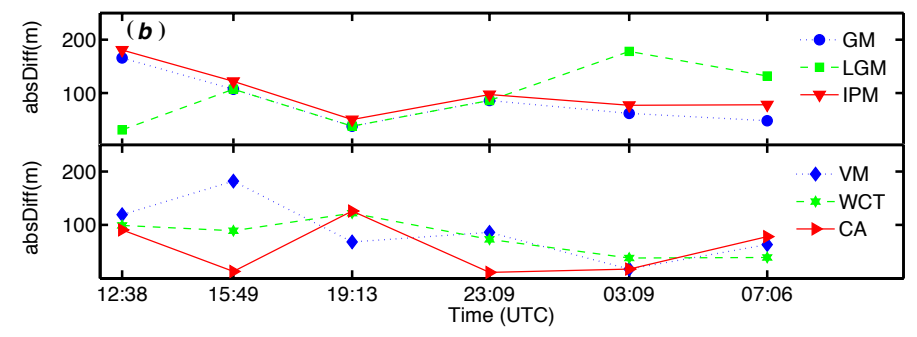

710 Figure 7. (a) and (b) are the same as Fig. 2a and $2 \mathrm{~b}$ of the manuscript, respectively, but

711 during the intensive radio soundings period corresponding to DD conditions.

712

713

714

715

716

717

718

719

720

721

722

723

724

725

726

727 


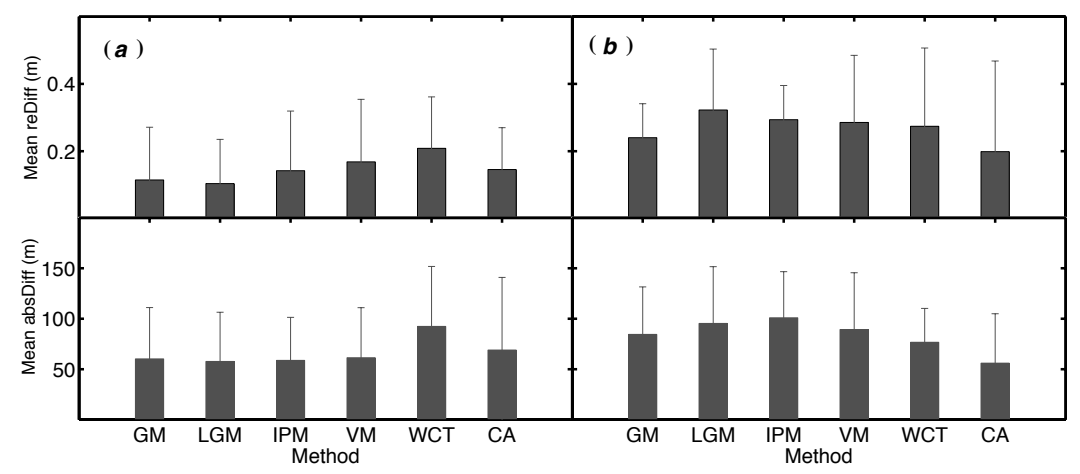

729 Figure 8. The same as Fig. 5, but for the aerosol conditions examined in this work: (a)

$730 \quad$ ND period and (b) DD period.

731

732

733

734

735

736

737

738

739

740

741

742

743

744

745

746

747 


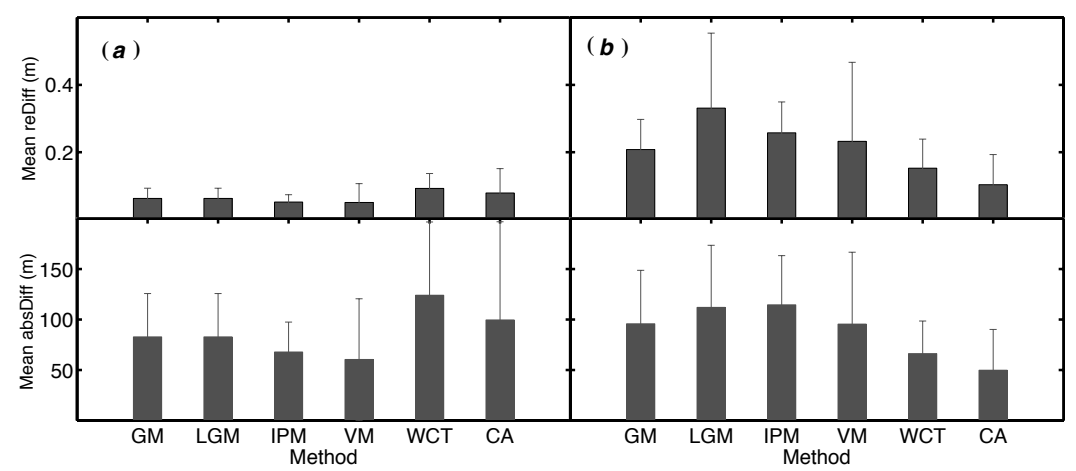

748

749 Figure 9. The same as Fig. 8 but considering in the analysis only the retrieved ABL

750 heights greater $300 \mathrm{~m}$.

751

752 